\title{
Vagueness: Subvaluationism
}

\author{
Pablo Cobreros \\ pcobreros@unav.es \\ A Philosophy Compass paper*
}

January 23, 2013

Supervaluationism is a well known theory of vagueness. Subvaluationism is a less well known theory of vagueness. But these theories cannot be taken apart, for they are in a relation of duality that can be made precise. This paper provides an introduction to the subvaluationist theory of vagueness in connection to its dual, supervaluationism.

A survey on the supervaluationist theory can be found in the Compass paper of Keefe (2008); our presentation of the theory in this paper will be short to get rapidly into the logical issues.

This paper is relatively self-contained. A modest background on propositional modal logic is, though not strictly necessary, advisable. The reader might find useful the Compass papers Kracht (2011) and Negri (2011) (though these papers cover issues of more complexity than what is demanded to follow this paper).

\section{Introduction. Vagueness and symmetry}

Some people are thin and some people are not. Some people, though, are not clearly thin and not clearly not thin (and this unclarity does not depend, it seems, on a lack of knowledge of the relevant measures of the people in question or on a failure of linguistic competence). Super and subvaluationism are theories of vagueness according to which this unclarity bears a direct

${ }^{*}$ I would like to thank the editors Kathrin and Peter and an anonymous reviewer of Philosophy Compass for suggestions to improve the final version. Further thanks go to my colleagues Paul Egré, David Ripley and Robert van Rooij and the Australian philosopher and paraconsistent logician Dominic Hyde for discussion and comments at different stages. Thanks also to Pablo Visiers. This work was done with support from the project 'Borderlineness and Tolerance' (FFI2010-16984), Ministerio de Economía y Competitividad, Government of Spain. 
connection to the meaning of 'thin'.

Here is a brief argument to the effect that vagueness requires a revision of classical semantics. Suppose that it is unclear (in the rough sense explained above) whether Tim is thin. Then competent speakers manifest a certain kind of symmetry in their dispositions to assert and deny the sentences 'Tim is thin' and 'Tim is not thin', so that they will generally refuse to assert one and deny the other. According to classical semantics, one sentence is true and the other is false; but any such truth-value assignment would introduce an asymmetry where our use (seemingly) sees none. Thus neither sentence is true (supervaluationism). Or both sentences are true (subvaluationism) ${ }^{1}$

A super- and sub-valuationist semantics (an s'valuationist semantics, for short) is a set of classical assignments. The distinctive feature of each theory is the notion of truth at play. According to supervaluationism a sentence is true in an interpretation just in case it is true in every classical assignment in that interpretation (that is, "truth is supertruth"). According to subvaluationism a sentence is true in an interpretation just in case it is true in some classical assignment in that interpretation (that is, "truth is subtruth"). A sentence is neither supertrue nor superfalse in an interpretation just in case it is both subtrue and subfalse in that interpretation. Examples like that of 'Tim is thin' are supposed to show that there are such sentences.

The subvaluationist theory applied to vagueness was first introduced in Hyde (1997). Subvaluationist logic, as we shall see, is a kind of paraconsistent logic. The idea that vagueness should be treated paraconsistently seems to go back to authors in the the Hegelian and Marxist tradition (without formal developement, though), Jaskowsky (1969), Halldén (1949) and, in more recent years, the Brazilian school on paraconsistent logic and Australian non-classical logicians like Priest and Routley (more about this in (Hyde, 2007, 94)). Discussion on Hyde's 1997 paper might be found in Akiba (1999), Beall and Colyvan (2001) (responses in Hyde (1999) and Hyde (2001)) and Odrowaz-Sypneiwska (1999). Formal development of subvaluationism received treatment prior to Hyde's paper in the work of Varzi (see Varzi (1997) and references to previous work in that paper). See Ripley (2013) for a recent discussion on super- and subvaluationism (that paper, though, argues for a truth-functional alternative, the paraconsistent LP). Hyde and Colyvan (2008) show that many prima-facie plausible arguments against the prospects of a paraconsistent explanation of vagueness are ultimately not compelling.

Most of the discussion in this paper takes a semantic perspective. The Appendix contains a particular proof-theory to deal with the logics discussed

\footnotetext{
${ }^{1}$ See, however, (Williamson, 1994, 205-9) for a defense of classical semantics in borderline cases in connection to this argument.
} 
in the paper. The reader should bear in mind that super- and sub- valuationist logics are sensitive to the choice of language and logical framework (where by logical framework we mean, roughly, the choice of what kind of elements might appear as premises and conclusions in a statement of consequence; see (Humberstone, 2011, 1.12)). We will begin from the most basic logical framework and language $\left(\mathrm{Fmla}, \mathcal{L}_{\mathrm{CPL}}\right)$ and end up with the more general setting (Set-Set, $\mathcal{L}_{\mathrm{MPL}}$ ).

\section{Super Classical and Sub}

\subsection{Logical consequence and duality}

A logical (propositional) language $\mathcal{L}$ is a set of expressions (the formulas of $\mathcal{L})$ which are either propositional variables or formulas constructed out of formulas with the aid of logical connectives. An interpretation for a language $\mathcal{L}$ is a function assigning truth-values to the formulas of $\mathcal{L}$. Depending on the kind of interpretation we are dealing with there might be several candidates to occupy the role of truth in that interpretation (think, for example, in a semantics with three values: $1, \frac{1}{2}$, 0 ; we can consider that being true is taking value 1 , or, alternatively, taking a value greater than 0 ).

Suppose that we have a language $\mathcal{L}$ and interpretation $\mathbb{I}$ for $\mathcal{L}$, and a notion of truth-in- $\mathbb{I}$ (for a formula of $\mathcal{L}$ ) true. Then, given sets $\Gamma, \Delta$ of formulas of $\mathcal{L}$ we say:

Definition 2.1.1 (Abstract logical consequence).

$\Delta$ is a logical consequence of $\Gamma$, written $\Gamma \vDash \Delta$, just in case there is no $\mathbb{I}$ such that

for all $A \in \Gamma \mathbb{I}(A)=$ true and for all $B \in \Delta \mathbb{I}(B) \neq$ true

This definition is given in what we will call "the Set-Set framework" (multiple-premises, multiple-conclusions). In addition to this, we will also consider the frameworks Set-Fmla (multiple-premises, single-conclusion) and Fmla-Set (single-premise, multiple-conclusions). These can be viewed as particular cases of the Set-Set framework: $\Gamma \vDash A$ is $\Gamma \vDash\{A\}$ and $A \vDash \Delta$ is $\{A\} \vDash \Delta$.

A definition of logical consequence like Definition 2.1.1 above intends to capture the idea of (necessary) preservation of truth, since it tells us that (in every interpretation) if all premises are true, some conclusions are true. We can reverse the point of view and think on logical consequence as backwards falsehood preservation: if all conclusions are false then some premises are false. 
Necessary preservation of truth and backwards falsehood preservation amount to the same thing whenever (for a given notion of logical consequence) falsity $=$ untruth. This fact is reflected, in terms of inferences, in the following equivalence,

$$
\Gamma \vDash \Delta \Longleftrightarrow \neg(\Delta) \vDash \neg(\Gamma) \quad \text { (where: } \neg(\Sigma)=\{\neg \sigma \mid \sigma \in \Sigma\} \text { ) }
$$

When, given a notion of logical consequence, falsity $\neq$ untruth the above equivalence no longer holds. Still, the equivalence will hold between two notions of logical consequence in which the notion of truth preserved is each other's dual (in general, two sentential operators $\oplus$ and $\otimes$ are duals just in case, $\oplus A$ is equivalent to $\neg \otimes \neg A$; like, for example, ' $\forall$ ' and ' $\exists$ '). We will officially say, then,

Definition 2.1.2 (Duality). $\vDash_{x}$ and $\vDash_{y}$ are duals just in case

$$
\Gamma \vDash_{x} \Delta \Longleftrightarrow \neg(\Delta) \vDash_{y} \neg(\Gamma)
$$

The idea of different dual consequence relations emerges every time falsity $\neq$ untruth. So super- and subvaluationist consequence are duals (as it will be pointed out after the formal definitions) but the same goes, for example, for K3 and LP. The interest of this connection is that it enables us to conclude facts about one consequence relation from facts about the other in dual form.

\subsection{The classical propositional language $\mathcal{L}_{\mathrm{CPL}}$}

In this section we discuss the logical relations between supervaluationist, subvaluationist and classical logic for the classical propositional language.

\subsubsection{Language and semantics}

A classical propositional language includes a denumerable set of propositional variables: $A, B, C \ldots$ and the set of constants: $\wedge, \vee, \neg, \supset$ to form complex sentences of the form $A \supset A, \neg(A \vee(B \wedge \neg C))$ etc. We call this language $\mathcal{L}_{\mathrm{CPL}}$.

A classical interpretation for $\mathcal{L}_{\mathrm{CPL}}$ is a function $\mathbb{I}$ from the set propositional variables to $\{1,0\}$. Given a classical interpretation, its extension is the function from the set of formulas of $\mathcal{L}_{\mathrm{CPL}}$ to $\{1,0\}$ satisfying the conditions: $\mathbb{I}(\neg A)=1$ iff $\mathbb{I}(A)=0, \mathbb{I}(A \wedge B)=1$ iff $\mathbb{I}(A)=\mathbb{I}(B)=1$ etc. By a classical interpretation we will generally mean an assignment of 1's and 0's to propositional variables together with the corresponding extension. 
An s'valuationist interpretation for $\mathcal{L}_{\mathrm{CPL}}$ is a collection $\mathbb{I}_{S}$ of classical interpretations: $\left\{\mathbb{I}^{\prime}, \mathbb{I}^{\prime \prime} \ldots\right\}$. Given an s'valuationist interpretation $\mathbb{I}_{S}$ for $\mathcal{L}_{\mathrm{CPL}}$ we say that a formula $A$ is supertrue just in case for all $\mathbb{I} \in \mathbb{I}_{\mathrm{S}}, \mathbb{I}(A)=1$ and that $A$ is subtrue just in case there is at least one $\mathbb{I} \in \mathbb{I}_{\mathrm{S}}$ such that $\mathbb{I}(A)=1$.

Following the schema in Definition 2.1.1, we define three distinct notions of logical consequence:

$\Delta$ is a classical consequence of $\Gamma$, written $\Gamma \vDash_{C L} \Delta$, just in case there is no II such that

for all $A \in \Gamma \mathbb{I}(A)=1$ and for all $B \in \Delta \mathbb{I}(B)=0$

$\Delta$ is a supervaluationist consequence of $\Gamma$, written $\Gamma \vDash_{\mathrm{SpV}} \Delta$, just in case there is no $\mathbb{I}_{S}$ such that

for all $A \in \Gamma$ and all $\mathbb{I} \in \mathbb{I}_{S} \mathbb{I}(A)=1$ and

for all $B \in \Delta$ there is a $\mathbb{I} \in \mathbb{I}_{\mathbf{S}} \mathbb{I}(B)=0$

$\Delta$ is a subvaluationist consequence of $\Gamma$, written $\Gamma \vDash_{\mathrm{SbV}} \Delta$, just in case there is no $\mathbb{I}_{S}$ such that

for all $A \in \Gamma$ there is a $\mathbb{I} \in \mathbb{I}_{\mathrm{S}} \mathbb{I}(A)=1$ and

for all $B \in \Delta$ and all $\mathbb{I} \in \mathbb{I}_{\mathrm{S}} \mathbb{I}(B)=0$

Though the formal definitions are a bit intricate, the informal reading is easy: $\Gamma \vDash_{\mathrm{SbV}} \Delta$ just in case there's no s'valuationist interpretation where all premises are subtrue while no conclusion is subtrue. Note that duality between $\vDash_{\mathrm{SpV}}$ and $\vDash_{\mathrm{SbV}}$ follows from these definitions:

$$
\Gamma \vDash_{\mathrm{SpV}} \Delta \Longleftrightarrow \neg(\Delta) \vDash_{\mathrm{SbV}} \neg(\Gamma)
$$

Informally, for any interpretation, 'if all the gamma's are supertrue, then some of the deltas are supertrue' is equivalent (by contraposition) to 'if all the deltas are subfalse, then some of the gamma's are subfalse'.

\subsubsection{Comparisons}

Consider the following facts:

Fact 2.2.1.

(i) $\Gamma \vDash_{\mathrm{Sp} \vee} A$ iff $\Gamma \vDash_{\mathrm{CL}} A$ (dually: $A \vDash_{\mathrm{Sb} \vee} \Gamma$ iff $A \vDash_{\mathrm{CL}} \Gamma$ ) 
Table 1: Relations on $\mathcal{L}_{\mathrm{CPL}}$

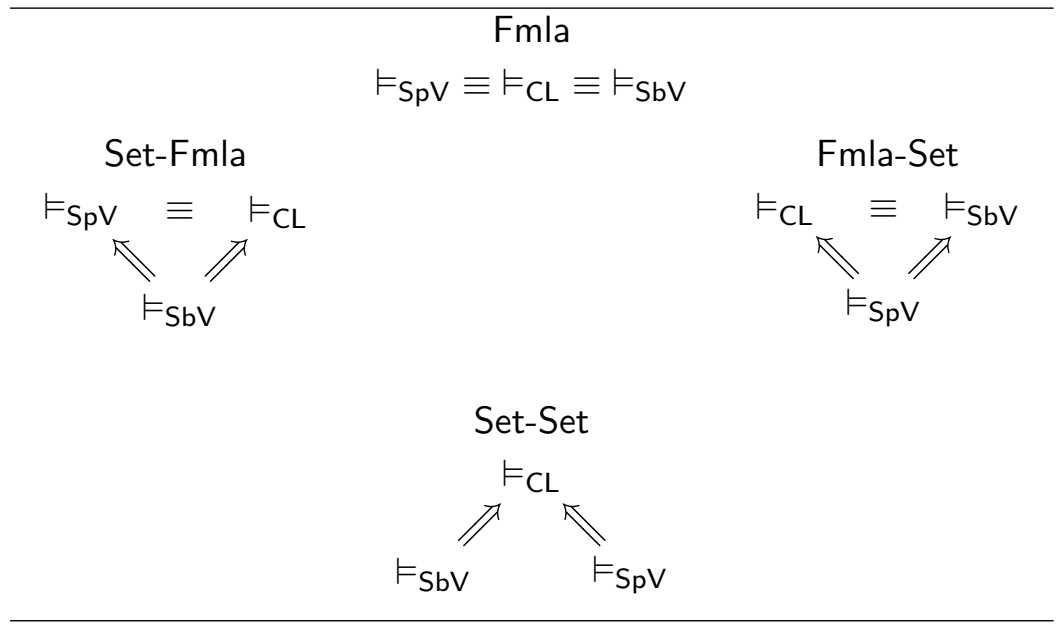

(ii) $\Gamma \vDash_{\mathrm{CL}} \Delta \not \Gamma \vDash_{\mathrm{SpV}} \Delta$ (dually: $\left.\Gamma \vDash_{\mathrm{CL}} \Delta \not \Gamma \vDash_{\mathrm{SbV}} \Delta\right)$

Comment 2.2.1. A paraconsistent logic is one in which the inference of explosion $(A, \neg A \vDash B)$ fails. Dually, a paracomplete logic is one in which the inference of implosion $(B \vDash A, \neg A)$ fails. Some paracomplete logics agree with classical logic "on the left" (for example, $\Gamma \vDash_{\mathrm{K} 3}$ just in case $\Gamma \vDash_{\mathrm{CL}}$, where ' $\Gamma \vDash$ ' means ' $\Gamma$ is not satisfiable') and, dually, some paraconsistent logics agree with classical logic "on the right" $\left(\vDash_{\mathrm{LP}} \Gamma\right.$ just in case $\left.\vDash_{\mathrm{CL}} \Gamma\right)$. Supervaluationism is a paracomplete logic and agrees with classical logic on the left. But fact (i) shows that (in $\mathcal{L}_{\mathrm{CPL}}$ ) the agreement extends to the Set-Fmla framework (see (Keefe, 2000, 175-6)). Dually, subvaluationism is a paraconsistent logic in agreement with classical logic in the Fmla-Set framework.

Comment 2.2.2. Fact (ii) holds because although implosion: $\vDash\{A, \neg A\}$ is classically valid, it is not valid according to supervaluationism. Dually, although explosion: $\{A, \neg A\} \vDash$ is classically valid, it is not valid according to subvaluationism.

Note, however, that from fact (i) it follows that $\vDash_{S p V}$ and $\vDash_{S b V}$ agree with $\vDash_{\mathrm{CL}}$ on the set of valid and unsatisfiable sentences. This fact, together with the observation just made about implosion and explosion shows that $\vDash_{\mathrm{SpV}}$ $/ \vDash_{\mathrm{SbV}}$ are weakly paracomplete / paraconsistent logic: ${ }^{2}$ in the sense that although implosion is not valid, $A \vee \neg A$ remains $\vDash_{\mathrm{SpV}}$-valid (and although explosion is not valid, $A \wedge \neg A$ remains $\vDash_{\mathrm{SbV}}$-unsatisfiable). Another way to

\footnotetext{
${ }^{2}$ See (Hyde, 2007, 75-6). Hyde credits this terminology to Arruda (1989).
} 
look at this peculiarity is noticing that the following is not generally valid in either $\vDash_{\mathrm{SpV}}$ and $\vDash_{\mathrm{SbV}}$ :

Conjoining premises, disjoining conclusions: $\Gamma \vDash \Delta \Leftrightarrow \Gamma^{\prime} \vDash \Delta^{\prime}$

(where $\Gamma^{\prime}$ comes from $\Gamma$ by possibly conjoining some of its members and $\Delta^{\prime}$ from $\Delta$ by possibly disjoining some of its members)

In other words, $\vDash_{\mathrm{Sp}}$ does not retain the standard connection between disjunction and the comma on the right, $\vDash_{S b V}$ does not retain the standard connection between the conjunction and the comma on the left. For this reason it is also said that these logics are not truth-functional. The relations between $\vDash_{\mathrm{SpV}}, \vDash_{\mathrm{CL}}$ and $\vDash_{\mathrm{SbV}}$ in $\mathcal{L}_{\mathrm{CPL}}$ are summarized in Table 1 .

Comment 2.2.3. In the philosophical the literature on vagueness, the sorites paradox is presented in at least two dresses. First, as an argument involving a universally quantified statement (over a suitable sorites series) like,

(U) $\forall x(x$ is bald $\supset x+1$ is bald $)$.

Second, as an argument involving instances of (U):

(1) (0) is bald $\supset(1)$ is bald

(2) (1) is bald $\supset(2)$ is bald

(n) $(n-1)$ is bald $\supset(n)$ is bald.

Due to the "weak" paraconsistency of subvaluationism, its response to the sorites paradox varies depending on the formulation of the argument. For the first formulation, the argument is valid but unsound (there is no precisification making true the premise (U) and so that premise is not even subtrue). For the second formulation, all the premises are subtrue (somewhere in the middle, some conditionals are both subtrue and subfalse), but the argument is not valid, since modus ponens might fail for the subvaluationist material conditional (particularly for a conditional that is both subtrue and subfalse). The response to the first formulation aligns with classical logic, the response to the second formulation aligns with paraconsistent logics (see (Dietz, 2011, sec. 5) for non-classical solutions to the sorites).

Some authors (Keefe, 2000, 199) have emphasized that this asymmetry in the treatment of sorites arguments is a very unappealing feature of the subvaluationist theory (after all, the different arguments seem to report the same sort of problem). At first sight this argument seems to provide convincing evidence for the supervaluationist theory. When we inspect it with more care, however, one can see that it draws heavily on the supposed 
naturalness of the Set-Fmla framework. Since the supervaluationist accepts the negation of the main premise but rejects each instance (Keefe, 2008, 316), we have that, in the following formulation of the argument:

$$
\exists x\left(P x \wedge \neg P x_{+1}\right) \vDash\left\{P a_{1} \wedge \neg P a_{2}, \quad P a_{1} \wedge \neg P a_{2}, \ldots, P a_{n-1} \wedge \neg P a_{n}\right\}
$$

the supervaluationist endorses the idea that the argument is not valid. Second, there are some reasons to prefer the "invalid-argument" response. In order to address a paradox, we are supposed to explain, not just where the argument goes wrong, but why it looks to be fine. In particular, regarding sorites arguments, we are supposed to explain why the main premise looks so appealing in the first place (what Fara (2000) calls the "psychological question"). The "valid-but-unsound" response tends to address this issue claiming that we somehow confuse the (untrue) main premise of the argument with some (true) weakening of it (like ' $\forall x[x$ is definitely bald $\supset x+1$ is not definitely not bald]'). But it is far from clear that ordinary speakers are confused in the proposed way and, in that case, the psychological question remains unaddressed. The subvaluationist solution to the paradox (in its second formulation) has the advantage of holding that all the conditionals in the argument are actually true. This, of course, depends on the failure of modus ponens as a valid rule of inference. Whether one think this is a high price to pay will depend on one's appeal for truth-value gluts (or dialetheism in general). Hyde explains that under the view, commonly associated to supervaluationism (see, for example, (Fine, 1975, 282)), according to which vagueness is a species of ambiguity, the subvaluationist response to the paradox (second formulation) is that the sorites is a case of fallacy of equivocation (Hyde, 1997, 649-50).

Comment 2.2.4. (For the $\mathcal{L}_{\mathrm{CPL}}$ language) supervaluationism coincides with classical logic in the Set-Fmla framework. Dually, subvaluationism coincides with classical logic in the Fmla-Set framework. Neither logic, however, is classical in the Set-Set framework. Is any logic more classical than the other? Keefe claims that the Set-Fmla framework is more natural than the Set-Set framework:

But we do not use multiple-conclusion arguments in ordinary life and it is reasoning in vague natural language that is in question. Multiple-conclusion logic is not intended as a logic of ordinary arguments (its study primarily has the role of providing a system for elegant analogies with multiple-premise logics) and its theses are questionably included among the body of 'traditional' logic. Keefe, 2000, 198, note 24)

I think Keefe's claim is disputable. After all, the use of a formal language and semantics (be it classical or otherwise) is already an abstraction from 
everyday reasoning, and the use of a Set-Set framework might be "natural" within that level of abstraction, given some advantages in theorising about everyday reasoning (Hyde's arguments for the "naturalness" of the Set-Set framework go along these lines (Hyde, 2010, 394-396)).

Comment 2.2.5. There is much discussion on whether the failure of truthfunctionality is really an advantage of these theories. We won't say more about it here, but see the discussion in (Keefe, 2000, 181-188) and (Hyde, 2010, 401-403).

\subsection{The modal propositional language $\mathcal{L}_{\mathrm{MPL}}$}

The target of a theory of vagueness, particularly of theories such as supervaluationism and subvaluationism, is to provide an analysis of the notion of a borderline case. It is natural, therefore, to consider a language extending $\mathcal{L}_{\mathrm{CPL}}$ for this purpose. This language will contain a definiteness operator such that $a$ is a borderline case of a predicate $P$ just in case it is neither definitely the case that $a$ is $P$ nor definitely the case that $a$ is not $P$.

As we did before, we want to explore the relations between supervaluationist, subvaluationist and classical logic for different logical frameworks, this time for a language containing a definitely operator. But there is a difficulty here. Classical logic is supposed to be a logic governing languages like $\mathcal{L}_{\mathrm{CPL}}$ or, perhaps, augmented with quantifiers but not for languages containing an operator for definiteness. In this respect, saying that some logic is "classical logic" when the language under consideration involves such non-classical notions is an abuse of terminology. Nevertheless, there is a wide agreement in the literature on vagueness that, in the present context, "classical logic" for a language with definiteness refers to something like a normal modal logic. There are several reasons for this. The most relevant, perhaps, that in a theory like epistemicism, committed to classical semantics and logic, the analysis of definiteness is provided in terms of a normal modal logic (see, for example, (Williamson, 1994, Ch. 8, Appendix)) in which there is minimal disruption of classical relations between inferences.

Our strategy regarding this issue will be the adoption of a more neutral terminology. Instead of "classical logic" and $\vDash_{C L}$ we will use "local consequence" and $\vDash_{\mathrm{L}}$; instead of "supervaluationist logic" and $\vDash_{\mathrm{SpV}}$ we will use "global consequence" and $\vDash_{\mathrm{G}}$ (the terminology of "local" and "global" will be made clear latter). Lastly, instead of "subvaluationist logic" and $\vDash_{\mathrm{SbV}}$ we will use (for lack of a better term) "subvaluationist consequence" and $\vDash_{\mathrm{s}}$. We will be mostly concerned with logical issues and so we can postpone the informal reading of these notions for the philosophical analysis of the results. For the same reason, we will use ' $\square$ ', instead of the more standard 
' $D$ ', for our definiteness operator.

\subsubsection{Language and semantics}

The language $\mathcal{L}_{\mathrm{MPL}}$ is an expansion of $\mathcal{L}_{\mathrm{CPL}}$ to a vocabulary including two new logical constants: $\square$ and $\diamond$. The formulas of $\mathcal{L}_{\mathrm{MPL}}$ can be, for example: $(\square A \supset A)$ and $\square(\square \square A \supset \diamond \neg \square \neg B)$. The three notions of logical consequence we are going to be concerned with have the same language and semantics, the difference comes, once again, from the definition of truth. The sort of interpretations we are going to work with are known as Kripke models.

An interpretation for the language $\mathcal{L}_{\mathrm{MPL}}$ is a triple $\langle W, R, \mathbb{I}\rangle$ where $W$ is a set of "points", $R$ is a relation in $W$ and $\mathbb{I}$ a function from $\langle w, A\rangle$ to $\{1,0\}$ (where $w \in W$ and $A$ is a propositional variable). We will write $\mathbb{I}_{w}(A)=1$ to mean that $\mathbb{I}$ assigns value 1 to the pair $\langle w, A\rangle$. Given a function $\mathbb{I}$ in an interpretation $\langle W, R, \mathbb{I}\rangle$, its extension is the function from the set of formulas of $\mathcal{L}_{\mathrm{MPL}}$ to $\{1,0\}$ satisfying the following conditions (for any given $w \in W$ ):

- $\mathbb{I}_{w}(A \wedge B)=1$ iff $\mathbb{I}_{w}(A)=\mathbb{I}_{w}(B)=1$

- $\mathbb{I}_{w}(\neg A)=1$ iff $\mathbb{I}_{w}(A)=0$

- $\mathbb{I}_{w}(\square A)=1$ iff for all $w^{\prime}$ if $w R w^{\prime}$ then $\mathbb{I}_{w}(A)=1$

(and similarly for the other connectives)

Comment 2.3.1. First note that our function $\mathbb{I}$ together with a given $w$ is a classical interpretation as defined in 2.1.1. Thus, an interpretation $\langle W, R, \mathbb{I}\rangle$ in which $\mathbb{I}$ is restricted to the classical vocabulary is a set of classical interpretations for $\mathcal{L}_{\mathrm{CPL}}$, that is, an s'valuationist interpretation (as defined in 2.1.1.) For this reason, this semantics meshes with the ideas in that section: we can think of $W$ as a collection of precisifications of the language. Second, $\square$ expresses definiteness (and so indefiniteness might be defined as $\neg \square A \wedge \neg \square \neg A$ ). The relation $R$ might be viewed as an "admissibility" relation between precisifications. Formally, this relation provides the means to define the semantics for logics weaker than S5. The logic of definiteness must be weaker than $\mathrm{S} 5$ if we want to make room for higher-order vagueness (for the possibility of borderline cases of borderline cases) since in S5 we have that $\vDash_{\mathrm{S} 5} \square A \vee \square \neg \square A$ (see (Williamson, 1999, 134)). Informally, we can think of $R$ as providing the set of precisifications that are deemed "reasonable" by the lights of a given precisification. We might have, for example, that according to precisification $w_{0}$ another precisification $w_{1}$ is reasonable, and we might have that $w_{1}$ deems as reasonable another precisification $w_{2}$ but that $w_{0}$ 
does not deem $w_{2}$ reasonable. Thus, according to this semantics, what is considered as definite might vary from precisification to precisification.

As we did in section 2.2.1 at least three notions of logical consequence can be defined depending on what counts as true in an interpretation.

$\Delta$ is a local consequence of $\Gamma$, written $\Gamma \vDash_{\mathrm{L}} \Delta$, just in case there is no interpretation $\langle W, R, \mathbb{I}\rangle$ and $w \in W$ such that

for all $A \in \Gamma \mathbb{I}_{w}(A)=1$ and for all $B \in \Delta \mathbb{I}_{w}(B)=0$

$\Delta$ is a global consequence of $\Gamma$, written $\Gamma \vDash_{\mathrm{G}} \Delta$, just in case there is no interpretation $\langle W, R, \mathbb{I}\rangle$ such that

for all $A \in \Gamma$ and all $w \in W \mathbb{I}_{w}(A)=1$ and

for all $B \in \Delta$ there is a $w \in W$ such that $\mathbb{I}_{w}(B)=0$

$\Delta$ is a subvaluationist consequence of $\Gamma$, written $\Gamma \vDash_{S} \Delta$, just in case there is no $\langle W, R, \mathbb{I}\rangle$ such that

for all $A \in \Gamma$ there is a $w \in W$ such that $\mathbb{I}_{w}(A)=1$ and

for all $B \in \Delta$ and all $w \in W \mathbb{I}_{w}(B)=0$

These definitions capture the ideas of preservation of local, global and subvaluationist truth, respectively. Note that $\vDash_{\mathrm{G}}$ and $\vDash_{\mathrm{S} \text {-consequence are }}$ duals as stated in section 2.1. Note also that, from these definitions, we might get different consequence relations by putting different restrictions on $R$. In the literature on vagueness it is standardly assumed that $R$ must be at least reflexive (this yields, for $\vDash_{\mathrm{L}}$, the consequence relation of normal modal logic $\mathbf{T}$ ). Whether we should impose further restrictions on $R$ depends on the informal reading of the semantics and issues connected to higher-order vagueness.

\subsubsection{Comparisons}

We showed in subsection 2.2.2 that super- and sub-valuationism and classical logic share the set of valid and unsatisfiable sentences over $\mathcal{L}_{\mathrm{CPL}}$. This relatively weak relation is already disrupted in $\mathcal{L}_{\mathrm{MPL}} . \vDash_{\mathrm{G}}$ agrees with $\vDash_{\mathrm{L}}$ on the set of valid formulas but not on the set of unsatisfiable formulas (as $A \wedge \neg \square A$ is locally, but not globally satisfiable). Dually, $\vDash_{\mathrm{S}}$ agrees with $\vDash_{\mathrm{L}}$ on the set of unsatisfiable formulas, but not on the set of valid formulas (as $\neg A \vee \square A$ is $\vDash_{\mathrm{S}}$-valid but not $\vDash_{\mathrm{L}}$ valid). Based on these failures we can already observe that some metainferences like contraposition and reductio won't generally hold for either $\vDash_{\mathrm{G}}$ and $\vDash_{\mathrm{S}}$ (consider $A \wedge \neg \square A$ : that's globally unsatisfiable, but its negation is not globally valid). 
The situation does not improve by extending things to the left (allowing multiple premises) or to the right (allowing multiple conclusions). $\vDash_{G}$ is strictly stronger than $\vDash_{\mathrm{L}}$ in the Set-Fmla framework, $\vDash_{\mathrm{S}}$ is strictly stronger than $\vDash_{\mathrm{L}}$ in the Fmla-Set framework. The following are some interesting inferences that are $\vDash_{\mathrm{G}}$ and $\vDash_{\mathrm{S}}$-valid but not $\vDash_{\mathrm{L} \text {-valid: }}$

Fact 2.3.1. [ $\square$-intro and $\diamond$-intro]

(a) $A \vDash_{\mathrm{G}} \square A$ but $A \not \nvdash_{\mathrm{L}} \square A$

(b) $A \vDash_{\mathrm{S}} \diamond A$ but $A \not \nvdash_{\mathrm{L}} \diamond A$

Attending to Fact 2.3.1 and noting that in $\mathcal{L}_{\mathrm{MPL}}$ we also have that $A, \neg A \nvdash_{\mathrm{S}}$ and $\nvdash_{\mathrm{G}} A, \neg A$, we arrive to the conclusion that in the Set-Set framework all three notions of logical consequence $\vDash_{\mathrm{G}}, \vDash_{\mathrm{L}}$ and $\vDash_{\mathrm{S}}$ are different and neither notion is contained in any of the others. A real mess! There is a way, however, to establish a link between $\vDash_{G}$ and $\vDash_{L}$ (and, by duality a link to $\vDash_{S}$ as well) making use of a property of modal logic known as the generated submodel property (( Humberstone, 2011,285$))$ credits this theorem to Segerberg (1971)).

Lemma 1 (Generated submodel). Let $\Gamma$ be a set of formulas of $\mathcal{L}_{\mathrm{MPL}}$ and $\langle W, R, \mathbb{I}\rangle$ an interpretation with a point $w_{0} \in W$ such that $\mathbb{I}_{w_{0}}(A)=1$ for all $A \in \Gamma$. Then, the interpretation $\left\langle W^{\prime}, R^{\prime}, \mathbb{I}^{\prime}\right\rangle$ defined:

- $W^{\prime}=\left\{w \mid \exists n\left(w_{0} R^{n} w\right)\right\}$ (' $R^{n}$ ' means ' $n$ steps along the relation $R$ ')

- $R^{\prime}$ and $\mathbb{I}^{\prime}$ are the restrictions of $R$ and $\mathbb{I}$ to $W^{\prime}$,

is such that $\mathbb{I}_{w_{0}}^{\prime}(A)=1$ for all $A \in \Gamma$

The intuitive idea of this lemma is as follows. Take a set of sentences $\Gamma$ that are true in a point $w_{0}$ in an interpretation $\langle W, R, \mathbb{I}\rangle$ : then you don't have to care about points $w$ such that $w_{0}$ is not connected to them by any number of steps along the accessibility relation (that is, points $w$ for which there's no $n$ such that $w_{0} R^{n} w$ ); what's true or false in $w_{0}$ does not depend on what's going on in those points. Thus, the interpretation resulting from chopping off these points is still an interpretation making true all of $\Gamma$ 's (see (Blackburn et al., 2001, 56) for more details on this).

Making use of this lemma we can connect $\vDash_{G}$ and $\vDash_{L}$ in the Set-Fmla framework. The connection is established within the language $\mathcal{L}_{\mathrm{MPL}}$ with the aid of the following definition:

Definition 2.3.1. [ $\square^{\omega}$-set]

Given a set $\Gamma$ of formulas of $\mathcal{L}_{\mathrm{MPL}}, \square^{\omega} \Gamma$ is the set $\left\{\square^{n} A \mid A \in \Gamma, n \in \omega\right\}$ 
That is, $\square^{\omega} \Gamma$ contains all the elements of $\Gamma$ plus all the elements of $\Gamma$ with one $\square$ attached, plus all the elements of $\Gamma$ with two $\square$ 's attached etc. We understand in a similar way the expression $\diamond^{\omega} \Gamma$, for any given $\Gamma$.

Theorem 1. $\left[\vDash_{G}-\vDash_{L}\right.$ connection]

$$
\Gamma \vDash_{\mathrm{G}} A \Longleftrightarrow \square^{\omega} \Gamma \vDash_{\mathrm{L}} A
$$

Proof. ( $\Longleftarrow)$ Assume $\Gamma \nvdash_{\mathrm{G}} A$. Then there is an interpretation where all the $\gamma$ 's take value 1 everywhere and $A$ value 0 somewhere (say, at point $w_{0}$ ). This point shows that $\square^{\omega} \Gamma \nvdash_{\mathrm{L}} A$.

$(\Longrightarrow)$ Assume now that $\square^{\omega} \Gamma \nvdash_{\mathrm{L}} A$. Then there is an interpretation $\langle W, R, \mathbb{I}\rangle$ and $w_{0} \in W$ such that every member of $\square^{\omega} \Gamma$ takes value 1 at $w_{0}$ and $A$ takes value 0 at $w_{0}$. Note that the first conjunct guarantees that any formula in $\Gamma$ will be true in any point $w$ connected to $w_{0}$ in any number of $R$ steps. Thus, any formula in $\Gamma$ takes value 1 everywhere in the corresponding generated submodel, showing this way that $\Gamma \nvdash_{\mathrm{G}} A$.

Corollary 1.1. $A \vDash_{\mathrm{S}} \Gamma \Longleftrightarrow A \vDash_{\mathrm{L}} \diamond^{\omega} \Gamma$

It is possible to make use of this theorem to provide a proof theory for $F_{G}$ in the Set-Fmla framework (see Cobreros (2011b)). The resulting proof system can be "dualized" for $\vDash_{S}$ in the Fmla-Set framework. Unfortunately, the theorem does not extend (not at least in a straightforward way) to the Set-Set framework. Whether there's any way to establish a connection between $\vDash_{G}$ and $\vDash_{\mathrm{L}}$ in the Set-Set framework making use just of the $\mathcal{L}_{\mathrm{MPL}}$ language, I don't know yet (see, however, Fact 4.2.1 in the Appendix, where a connection is made making use of a symbol not in $\mathcal{L}_{\mathrm{MPL}}$ ).

Comment 2.3.2. Imagine a long enough sorites series for the predicate 'red'. There seems to be no sharp transition from the red objects to the non-red objects. The supervaluationist explains this fact saying that there's actually no sharp transition between these sort of objects: the definitely red objects and the definitely not red are separated by a "gap" (those that are neither definitely red nor definitely not red). In other words, the absence of such transition is guaranteed by the truth of the following gap principle:

$$
\text { (GP for } \Psi) \square \Psi(x) \supset \neg \square \neg \Psi\left(x^{\prime}\right)
$$

Now there seems to be no sharp transition from the definitely reds and the not definitely reds, nor from the definitely definitely reds to the not definitely definitely reds etc. and parsimony demands and equal explanation to these absence of transitions. Thus, the supervaluationist is committed to each of the following gap-principles (for each natural number $n$ ): 


$$
\text { (GP for } \left.\square^{n} \Psi\right) \square \square^{n} \Psi(x) \supset \neg \square \neg \square^{n} \Psi\left(x^{\prime}\right)
$$

Fara (2003) shows that a theory committed to the inference: $A \vDash \square A$ plus some minimal constraints on the modal treatment of $\square$ cannot endorse all these gap-principles for finite sorites series (Fara's argument is actually a proof that these principles are $\vDash_{\mathrm{G}}$-unsatisfiable for finite sorites series, see (Cobreros, 2011a, Appendix)). So supervaluationism is in trouble, a trouble known as 'the paradox of higher-order vagueness'. This sort of problem,

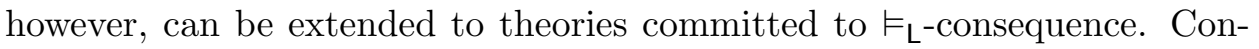
sider again theorem 1. If gap-principles are $\vDash_{\mathrm{G}}$-unsatisfiable then, by this theorem, the "absolute definitization" of those principles (the resulting of

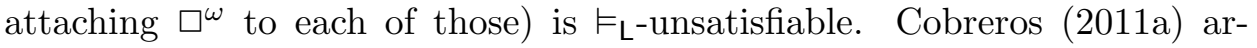
gues that such strengthening of gap-principles is actually compelling for any theory making essential use of definiteness. But the strengthening of the principles is $\vDash_{S}$-satisfiable (see Cobreros (2008) and Cobreros (2011b) for further discussion on logical consequence and gap-principles; see Zardini (2011) for a paradox of this sort requiring fewer assumptions).

\section{Conclusion}

In this paper we presented the subvaluationist theory in connection to its dual, the well-known supervaluationist theory. Despite this duality there are several arguments for one and against the other.

For the subvaluationist theory we have, at least, its endorsement of symmetry in borderline cases that allows us to assert both a sentence and its negation (rather than forcing silence), its solution to the sorites where all the conditionals come out true and it's ability to accept the absolute definitization of gap-principles. Against the subvaluationist theory we have, among other things (and assuming that classical logic is a default option), the failure of classicality in the Set-Fmla framework, its non-detachable material conditional and its non-uniform solution to different formulations of the sorites series.

Some philosophers take paraconsistency as sufficient evidence to disregard a theory. But full inspection of a theory will surely reveal more (about this and related theories) than a quick rejection. And the subvaluationist theory deserves, at the very least, that credit. 


\section{Appendix: Proof theory}

The previous section dealt with relations between super- sub- and classical logic (in $\mathcal{L}_{\mathrm{CPL}}$ and $\mathcal{L}_{\mathrm{MPL}}$ ) from a model theoretic perspective. But simple inspection on the semantic characterization of a consequence relation might not be enough to decide whether a statement of consequence holds. In this section we are concerned with ways to decide, for a given statement of consequence, whether that statement holds. Our system for this purpose is an adaptation of modal tableaux (that is, tableaux for $\vDash_{\mathrm{L}}$ ) to find proofs for either $\vDash_{G}$ or $\vDash_{S}$ in the Set-Set framework (this is our most general setting: we can straightforwardly adapt this system to either different logical frameworks or to the language $\mathcal{L}_{\mathrm{CPL}}$ ).

\subsection{Modal tableaux}

The tableaux method consists on a set of rules to construct a tree out of a list of formulas $A_{1}, \ldots A_{n}$. The rules tell us how to extend the tree chopping formulas in the initial list into more basic formulas until no further application of a rule is allowed (there's nothing left to chop). At that point the structure of the tree reveals whether the initial list of formulas is or is not satisfiable. Any question about logical consequence will be translatable into a question about the satisfiability of a certain set.

Let $\Gamma$ be a set of formulas of $\mathcal{L}_{\mathrm{MPL}}$. The nodes of our trees can be of two forms: either pairs of the form $\langle A, i\rangle$ where $A$ is a formula of $\mathcal{L}_{\mathrm{MPL}}$ and $i$ a natural number (representing a point in an interpretation) or something of the form $i r j$ (encoding that world $i$ accesses to world $j$ ). A branch in a tree is closed when there are two nodes of the form $\langle A, i\rangle$ and $\langle\neg A, i\rangle$ (NB: the ' $i$ ' must be the same) in that branch. A tree is closed when all its branches are closed. We say that $\Gamma \vdash \Delta$ just in case there is a closed tree for an initial list containing $\Gamma \cup \neg \Delta$. A detailed description of these trees for $\vDash_{\mathrm{L}}$ can be found in (Priest, 2008, ch. 1-3).

\subsection{Tableaux for $\vDash_{G}$ and $\vDash_{S}$}

Our target is adapting the method of tableaux for $\vDash_{G}$ and $\vDash_{S}$. We will make use of a universal modality.

Definition 4.2.1 (Universal modality). For any interpretation $\langle W, R, \mathbb{I}\rangle$ : $\mathbb{I}_{w}\left(\square_{u} A\right)=1$ just in case for all $w \in W: \mathbb{I}_{w}(A)=1$

Fact 4.2.1. $\Gamma \vDash_{\mathrm{G}} \Delta \Longleftrightarrow \square_{u} \Gamma \vDash_{\mathrm{L}} \square_{u} \Delta$

Proof. From definitions. 
Dually, we have that $\Gamma \vDash_{S} \Delta \Longleftrightarrow \diamond_{u} \Gamma \vDash_{\mathrm{L}} \diamond_{u} \Delta$. The tree rules for nodes of the form $\left\langle\square_{u} A, i\right\rangle$ and $\left\langle\diamond_{u} A, i\right\rangle$ work like the rules for $\square$ and $\diamond$ except that no mention of the accessibility relation is needed (see (Priest, 2008, 45)). We will say that $\Gamma \vdash_{\mathrm{G}} \Delta$ just in case there is a closed tree for $\square_{u} \Gamma \cup \neg \square_{u} \Delta$ and that $\Gamma \vdash_{s} \Delta$ just in case there is a closed tree for $\diamond_{u} \Gamma \cup \neg \diamond_{u} \Delta$.

Example 4.2.1. $A \vee B \nvdash_{\mathrm{G}} A, B$

Proof.

$$
\begin{gathered}
\square_{u}(A \vee B), 0 \\
\neg \square_{u} A, 0 \\
\neg \square_{u} B, 0 \\
\neg A, 1 \\
\neg B, 2 \\
A \vee B, 1 \\
A \vee B, 2
\end{gathered} \overbrace{A, 1 \quad B, 1}^{B, 2}
$$

Example 4.2.2. $A \supset B, A \nvdash_{\mathrm{S}} B$

$$
\begin{aligned}
& \text { Proof. } \\
& \diamond_{u}(A \supset B), 0 \\
& \diamond_{u} A, 0 \\
& \neg \diamond_{u} B, 0 \\
& \square_{u} \neg B, 0 \\
& A \supset B, 1 \\
& \text { A, } 2 \\
& \neg B, 1 \\
& \neg B, 2 \\
& \neg \widehat{A, 1 \quad B, 1} \\
& \otimes
\end{aligned}
$$

Example 4.2.3. $A \wedge \neg \square A \vdash_{\mathrm{G}} \emptyset$ 
Proof.

$$
\begin{gathered}
\square_{u}(A \wedge \neg \square A), 0 \\
A \wedge \neg \square A, 0 \\
A, 0 \\
\neg \square A, 0 \\
\diamond \neg A, 0 \\
0 r 1 \\
\neg A, 1 \\
A \wedge \neg \square A, 1 \\
A, 1 \\
\otimes
\end{gathered}
$$

The soundness and completeness for $\vDash_{\mathrm{L}}$ together with fact 4.2 .1 ensure that these tableaux are sound and complete for $\vDash_{G}$ and $\vDash_{S}$, respectively.

\section{References}

Akiba, K. (1999). On super- and subvaluationism: a classicist's reply to Hyde. Mind, 108(432):727-732.

Arruda, A. I. (1989). Aspects of the historical development of paraconsistent logic. In G. Priest, R. Routley and J. Norman (eds.) Paraconsistent Logic: essays on the inconsistent. Philosophia Verlag.

Beall, J. C. and Colyvan, M. (2001). Heaps of gluts and Hyde-ing the sorites. Mind, 110(438):401-408.

Blackburn, P., Rijke, M., and Venema, Y. (2001). Modal Logic. Cambridge University Press (Reprinted with corrections: 2004).

Cobreros, P. (2008). Supervaluationism and logical consequence: a third way. Studia Logica, 90(3):291-312.

Cobreros, P. (2011a). Paraconsistent vagueness: a positive argument. Synthese, 183(2):211-227.

Cobreros, P. (2011b). Varzi on supervaluationism and logical consequence. Mind, 120(479):833-43.

Dietz, R. (2011). Vagueness and indeterminacy. The Continuum Companion to Philosophical Logic, eds. L. Horsten and R. Pettigrew. Continuum.

Fara, D. G. (2000). Shifting sands: An interest-relative theory of vagueness. Philosophical Topics. Originally published under the name 'Delia Graff', 28(1):45-81. 
Fara, D. G. (2003). Gap principles, penumbral consequence and infinitely higher-order vagueness. In J. C. Beall (ed.) Liars and Heaps: New Essays on Paradox. Oxford University Press. Originally published under the name 'Delia Graff', pages 195-221.

Fine, K. (1975). Vagueness, truth and logic. Synthese, 30:265-300.

Halldén, S. (1949). The Logic of Nonsense. Uppsala: Uppsala Universitets Arksskrift.

Humberstone, L. (2011). The Connectives. MIT Press.

Hyde, D. (1997). From heaps and gaps to heaps of gluts. Mind, 106(424):641-660.

Hyde, D. (1999). Pleading classicism. Mind, 108(432):733-735.

Hyde, D. (2001). A reply to Beall and Colyvan. Mind, 110(438):409-411.

Hyde, D. (2007). Vagueness, Logic and Ontology. Ashgate.

Hyde, D. (2010). The prospects of a paraconsistent response to vagueness. In R. Dietz and S. Moruzzi (eds.) Cuts and Clouds. Oxford University Press.

Hyde, D. and Colyvan, M. (2008). Paraconsistent vagueness: Why not? Australasian Journal of Logic, 6:107-21.

Jaskowsky, S. (1969). Propositional calculus for contradictory deductive systems. Studia Logica, 24:143-57 (Originally published in 1948 in Polish in Studia Scientarium Torunensis, Sec. A 11: 55-77).

Keefe, R. (2000). Theories of Vagueness. Cambridge University Press.

Keefe, R. (2008). Vagueness: Supervaluationism. Philosophy Compass, $3: 315-324$.

Kracht, M. (2011). Technical modal logic. Philosophy Compass, 6:350-359.

Negri, S. (2011). Proof theory for modal logic. Philosophy Compass, 6:52338.

Odrowaz-Sypneiwska, J. (1999). Heaps and gluts: Paraconsistent logic applied to vagueness. Logic and Logical Philosophy, 7:179-193.

Priest, G. (2008). An Introduction to Non-Classical Logic: From If to Is. Cambridge University Press.

Ripley, D. (2013). Sorting out the sorites. In Berto, F., E. Mares, Paoli, F. and Tanaka, K. (eds.) Paraconsistency: Logic and Applications. Springer. 
Segerberg, K. (1971). An Essay in Classical Modal Logic. Filosofiska Studier, Uppsala.

Varzi, A. (1997). Inconsistency without contradiction. Notre Dame Journal of Formal Logic, 38:621-640.

Williamson, T. (1994). Vagueness. Routledge.

Williamson, T. (1999). On the structure of higher-order vagueness. Mind, 108(429):127-143.

Zardini, E. (2011). Higher-order sorites paradox. Journal of Philosophical Logic (forthcoming). 